\title{
A POSSIBLE CASE OF OEDEMATOUS FIBROMA WITH INFLAMMATORY CHANGES
}

\author{
BY \\ VIGGO ESKELUND \\ From the Institute of Pathological Anatomy, University of Copenhagen
}

With the advances made in lung surgery during the last decades, interest in benign tumours of the lung has greatly increased. In the past these tumours were usually seen at necropsy, but to-day, with improved diagnosis and extensive routine examinations for tuberculosis, they can be detected early enough to be treated by surgery. Surgical treatment is generally advisable, because it is nct possible to exclude malignant change with certainty-for example, in hamartoma.

The following case illustrates how an unusual benign tumour can cause difficulties in diagnosis.

\section{CASE RePORT}

The patient was a woman aged 52. At 29 years she had had pleurisy on the right side, and was admitted to hospital and later to a tuberculosis sanatorium. Since then she has been admitted to hospitals more than 20 times for disease of the right lung, which she believes originated in the pleurisy. In 1942 she was in a tuberculosis hospital in Copenhagen, where it was thought that her main symptom, dyspnoea, was caused by cystic lungs and a spontaneous pneumothorax. Screening of the lungs showed irregular translucent areas in the upper and middle lobes of the right lung with displacement of the mediastinum to the left (Fig. A). Puncture of the right pleural cavity showed a positive pressure, varying up to $14 \mathrm{~cm}$. of water, which fell rapidly, relieving the dyspnoea, but rose again after five to ten minutes.

At one time the patient had an excessive, foul-smelling sputum, particularly on movement. Tubercle bacilli were never found; she had never had a haemoptysis. Her main complaints were dyspnoea, both with exercise and at rest in a horizontal position, and an almost constant pain in the right side of the thorax. The pain felt "like a wound"; it was not aggravated by respiration or coughing. She also complained of oppressive pain in the precordial region, sometimes accompanied by palpitations. In December, 1947, she had a serious attack of dyspnoea with angina-like pain. She was admitted to Naestved Hospital, and the symptoms promptly disappeared after puncture of the pleural cavity and release of air from the pleural cavity. A cystic right lung with pneumothorax was diagnosed. The sedimentation rate was 88 on admission and fell to 17 . The Mantoux reaction was weakly positive. The electrocardiogram was normal.

She had been operated on for an ovarian tumour when she was young. She had never been pregnant; she reached the menopause at the age of 50 . At 51 she had "kidney trouble," with fever and frequent micturition. The symptoms disappeared after treatment with sulphonamides.

In September, 1948, she was admitted to the Department of Thoracic Surgery, Rigshospitalet. She was rather thin and tired. The temperature was normal. Auscultation revealed diminished respiration over the whole of the right side; there 


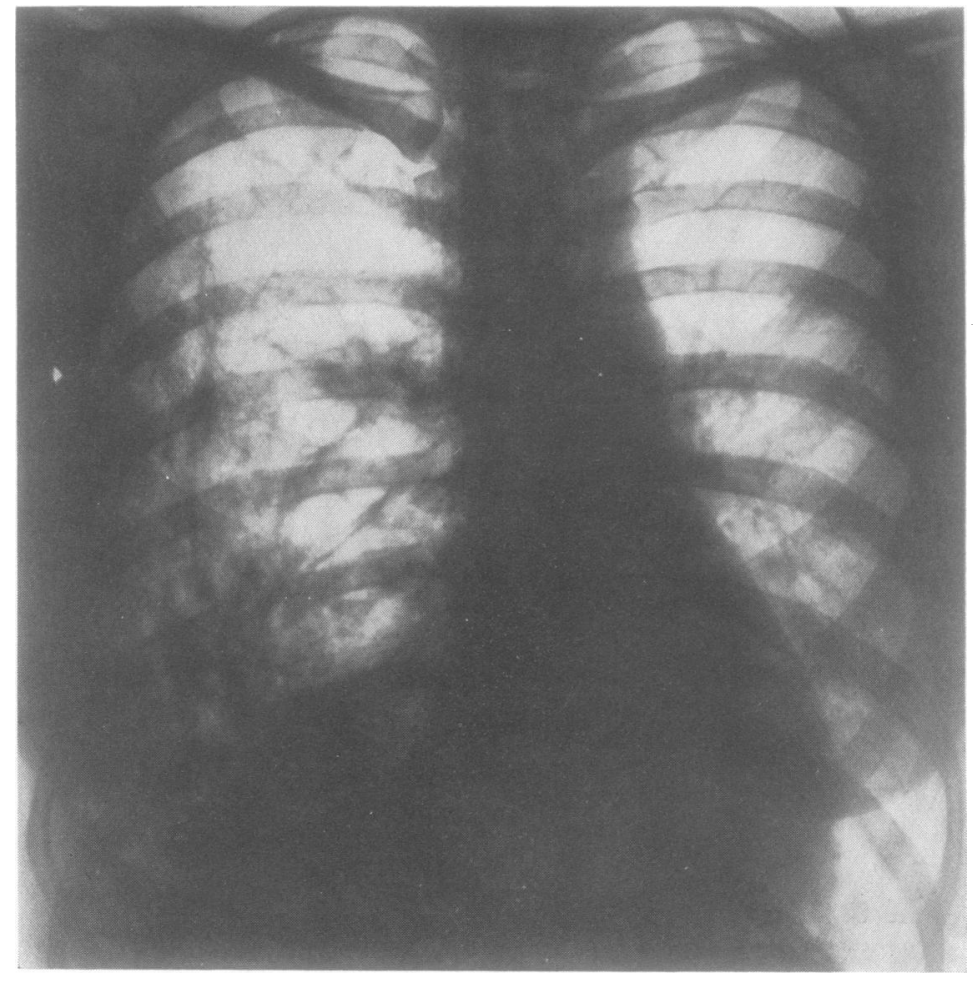

Fig. A

were no râles. Radiography (Figs. A and B) of the chest showed a slight distension of the right side with depression of the right diaphragm. The entire right lung showed an increase in the usual markings which was most marked towards the base, and in the lateral view appeared to consist of cystic translucencies of various sizes. No patches of infiltration or mottling were seen and there was no evidence of pneumothorax. The heart, which was probably rotated, the trachea, and the mediastinum were displaced to the left. The left lung was normal. A cystic right lung, due possibly to valve formations in the walls of the cysts, was diagnosed.

Other findings were: blood pressure $140 / 90$; sedimentation rate 44 (Westergren); serum protein level 8 ; haemoglobin $90 \%$; Wassermann reaction negative; urine normal; Mantoux reaction weakly positive.

Operation was performed on October 7, 1948, under general anaesthesia. This was intratracheal nitrous oxide-oxygen-ether, using a respirator. Access to the lung was obtained through a postero-lateral incision with resection of the fifth rib and division of the sixth and seventh ribs posteriorly. The lung was very adherent to the inside of the chest wall; pneumothoracic spaces and lung cysts were entered when the pleura was opened. The greater part of the lung had to be separated from the chest wall extrapleurally, and the pleura was opened when the hilum was reached. When the lung had been completely freed it was seen that the upper lobe occupied nearly the whole of the thoracic cavity. This lobe consisted of soft, spongy tissue containing a few large and many small cysts. The middle and lower lobes were atelectatic and pressed down on to the diaphragm. The interlobular space was easily entered; the hilus was dissected free and forceps were put on the arteries, veins, and 


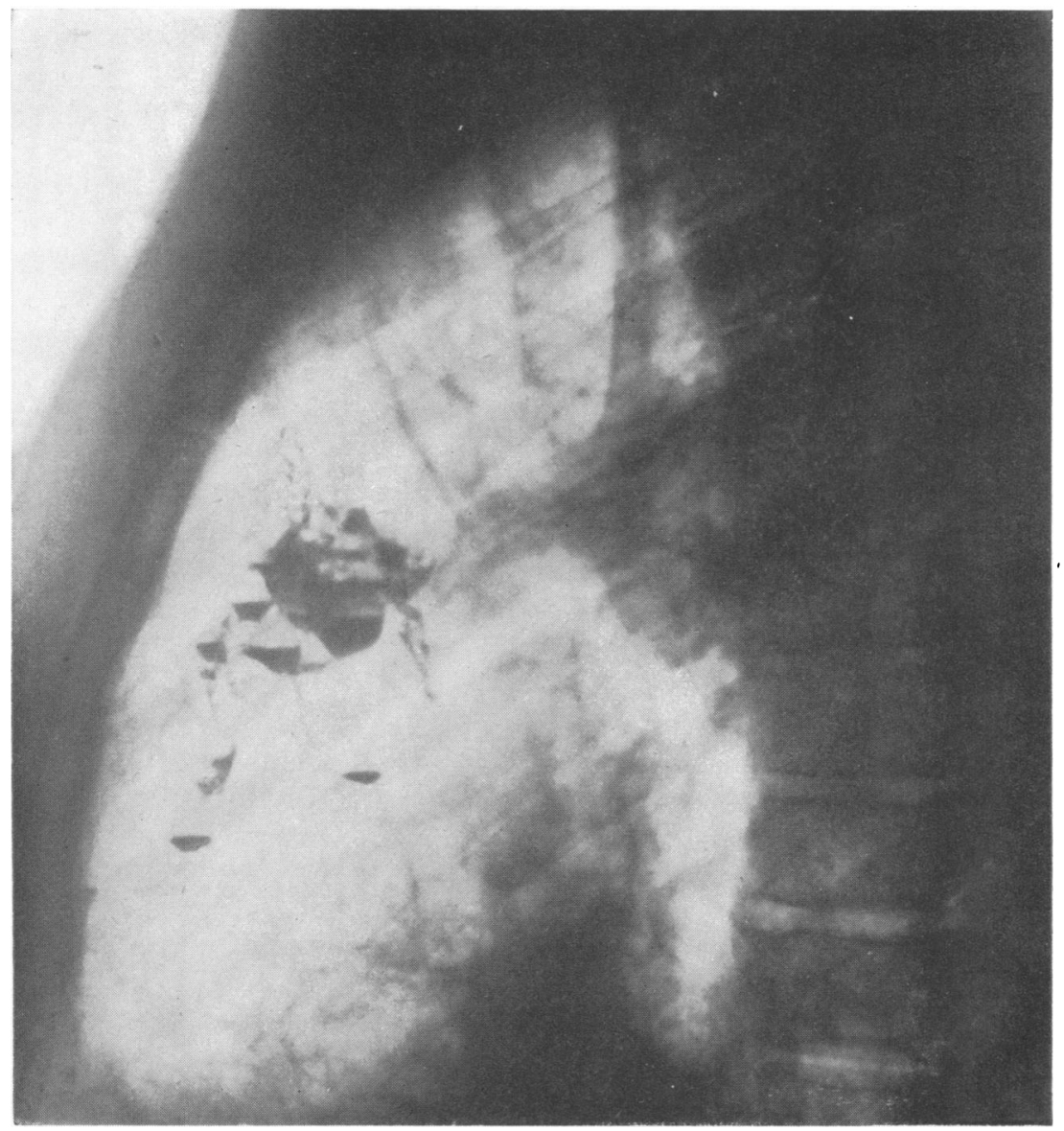

FIG. B

bronchus to the upper lobe. Double ligatures were put on the vessels and the bronchus was closed with silk sutures. It was then possible to inflate the middle and lower lobes. Although these together were not bigger than a grapefruit, and had correspondingly small vessels and bronchi, it was decided to preserve them. The phrenic nerve was resected; a pezzar catheter was inserted inferiorly and posteriorly and 200,000 units of penicillin were run into the thoracic cavity. The wound was closed with linen thread. During the operation transfusions amounting to $2,300 \mathrm{ml}$. in all were given. The drain was removed after 48 hours.

During the first ten days the temperature was rather high, swinging up to $40^{\circ} \mathrm{C}$.; afterwards it remained constant at about $38^{\circ} \mathrm{C}$. On the third day after operation the patient suddenly became severely shocked. The blood pressure was too low to be measured and there were long pauses in respiration. She recovered after a blood transfusion, and some tenacious plugs of sputum were removed by bronchoscopic aspiration. During the following period repeated pleural punctures, which released air and haemorrhagic fluid, were necessary. Penicillin was given, but was discontinued as the tongue became red and irritated. Injections of streptomycin were then given The patient's condition deteriorated : a wide-bore drainage tube was inserted into the 
FIG. 3

FIG. 1
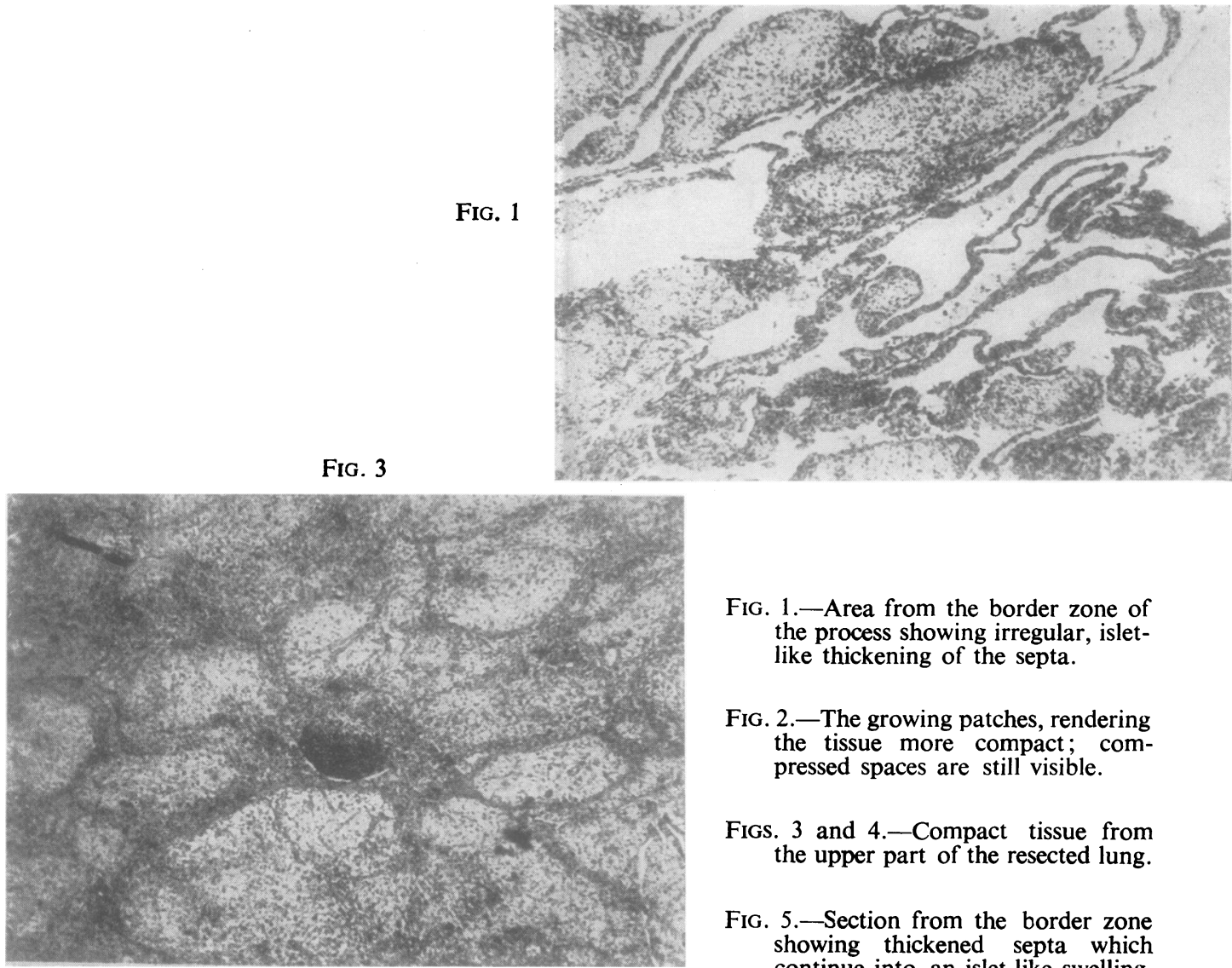

FIG. 1.-Area from the border zone of the process showing irregular, isletlike thickening of the septa.

FIG. 2.-The growing patches, rendering the tissue more compact; compressed spaces are still visible.

Figs. 3 and 4.-Compact tissue from the upper part of the resected lung.

FIG. 5.- Section from the border zone showing thickened septa which continue into an islet-like swelling.

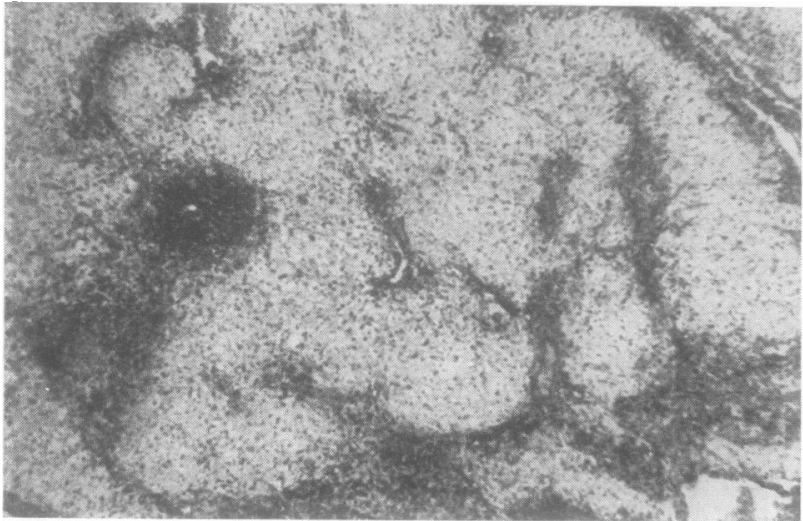

Fig. 6.-Loose tissue with capillaries and inflammatory changes, particularly in the periphery.

FIG. 7.-Fibrous streak in the upper part of the lung.

FIG. 8.-Concretions in compressed bronchioles.

FIG. 9.-Loose oedematous tissue with coarse collagenous fibrils.

FIG. 4

Fig. 2

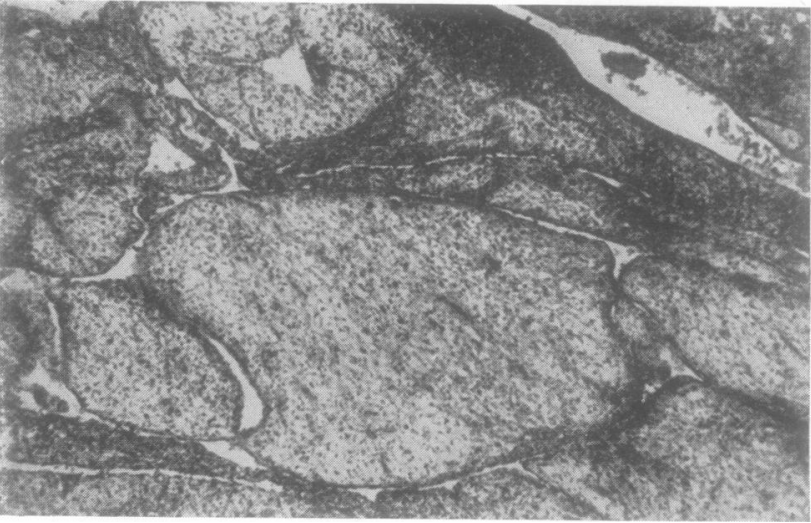




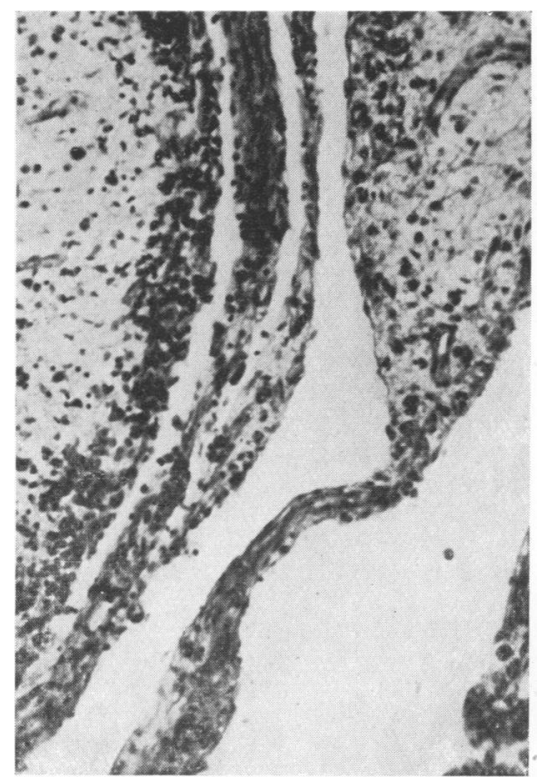

FIG. 5

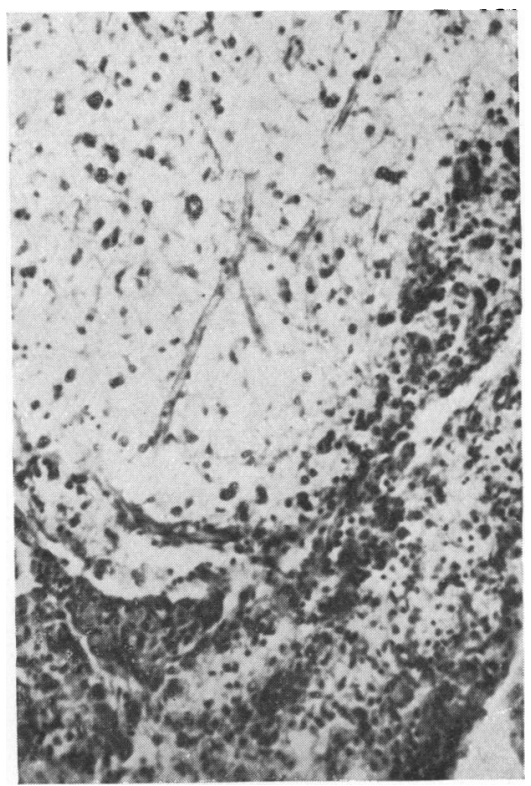

Fig. 6

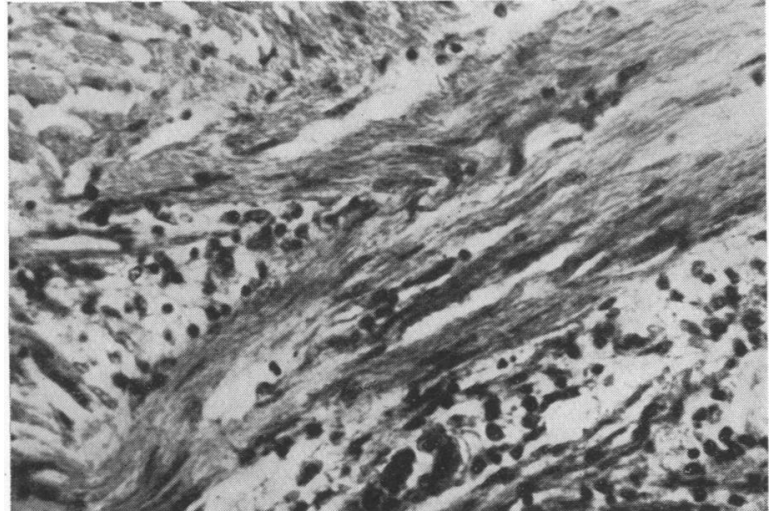

FIG. 7

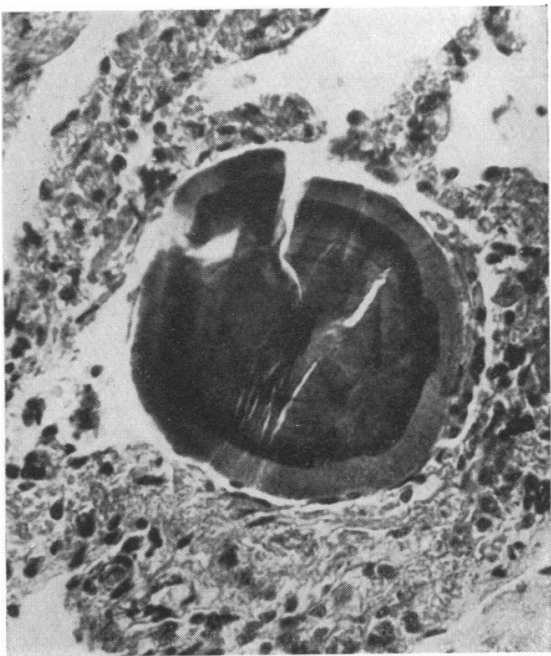

FIG. 8

FIG. 9

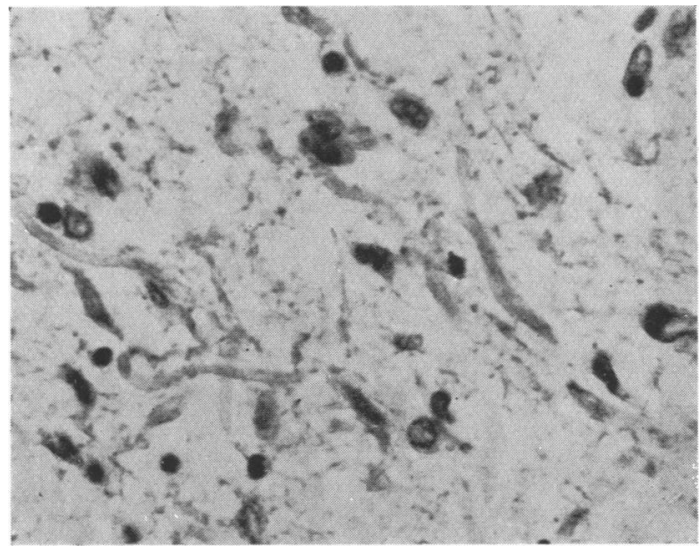


pleural cavity, and large quantities of foul-smelling, blood-stained fluid were drained off. Since then the general health of the patient has improved, but there is still some discharge from the drainage site.

\section{Pathology}

The resected lobe measured $25 \times 20 \times 5 \mathrm{~cm}$. The surface was very uneven. Section showed numerous cavities, varying from a few $\mathrm{mm}$. to $6 \mathrm{~cm}$. in diameter ; some contained a mucous fluid. The intervening tissue was whitish, soft, and nodular, the single lumps measuring up to $7 \mathrm{~cm}$. in diameter. Inferiorly in the lobe the lumps were small and widely spaced, whereas superiorly they were confluent and generally firmer. No normal lung tissue was found.

Microscopic examination (Figs. 1-9) of the tissue showed that it was made up of cells with oval nuclei and somewhat jagged protoplasm. The nuclei showed little variation and were small. In most places these cells were scattered in an oedematous matrix with coarse, wavy, and branched, or streak, arrangement of the collagenous fibrils. There were a number of spaces in the tissue, but in some places it was compact and lobules of varying size could be distinguished; these lobules were bounded indefinitely by streaks of varying thickness, rather rich in cells. In other places the lobules were bounded by narrow spaces with and without low cylindrical or cuboid epithelial cells. Cysts had formed where these spaces were widest. There were numerous large and small groups of lymphocytes, surrounded by infiltrations of plasma cells. There were no signs of acute inflammation and no reaction foci. Most of the groups of lymphocytes were near the cysts and spaces. In these spaces, which we considered to be rudimentary bronchioles and alveoli, some phagocytic cells and occasional foreign body giant cells containing vacuoles were seen. Concentric concretions were also found in some areas. The firmer parts in the upper region of the resected lung were richer in collagenous fibrils, which sometimes formed dense streaks. Irregular streaks of smooth muscular tissue were also present. There were many blood vessels, and the loose tissue contained a number of capillary vessels. Round the larger vessels there were compact streaks of collagenous connective tissue, radiating into the surrounding tissue. There were haemorrhages and clumps of haemosiderophages. There was no necrosis. There was a smooth transition from the loose oedematous tissue, which in places was markedly, and diffusely, infiltrated with plasma cells and lymphocytes, to the denser fibrous tissue. The border zone showed partly compressed alveolar septa of normal thickness, partly broad, thickened septa with loose oedematous stroma, and partly irregular thickening of the septa, forming islet-like patches of varying size. The inflammatory changes in these patches and thickened septa were usually localized to the superficial layers. In the surrounding compressed lung tissue there were a number of small concretions similar to those described above. There was no evidence of tuberculosis. Mucous stains showed no mucin.

\section{Discussion}

The radiographs and clinical findings excluded tuberculosis and syphilis, and the likely diagnosis seemed to us to be a "cystic lung." Among cystic formations of the lung we considered it possible to exclude dermoid cysts, hydatid cysts, and ectopic gastric cysts. There remained congenital cystic lung; in this condition the cysts are usually of bronchial origin; they may be single or multiple, localized or diffuse, 
unilateral or bilateral. They are found in persons of all ages. They are often complicated by inflammation and valvular pneumothorax formation, with compression and displacement of the mediastinum. Rupture of the cyst, or cysts, and pneumothorax formation with possible infection and pyopneumothorax are not uncommon. In the case described here there had been several episodes of valvular pneumothorax formation and febrile pulmonary inflammation. Clinically and pre-operatively, the only possible diagnosis was congenital cystic lung with complications. But the findings at operation and on histological examination showed this to be a rather unusual case. The most characteristic thing about this lesion was the invasive growth in the lung of oedematous fibroma-like tissue, which had converted the lung into areas of dense tissue of varying size. The irregular invasive growth in the border zone, with compression of the bronchi and alveoli, explained the frequent valve formation. The tissue showed rather marked chronic inflammatory changes, particularly localized to the remains of the alveolar cavities. The inflammatory changes were non-specific. The cavities in the lobe were either devoid of epithelium or lined with a low cylindrical or cuboid epithelium. In view of these inflammatory changes we considered the possibility of a progressive, interstitial inflammation, with a similar histological picture to that found in nasal polypi, caused by inflammation. Such an inflammatory process has, so far as can be ascertained, not been described previously. The macroscopic appearance of the tissue seemed to us to be most like a tumour. Further, the inflammatory changes were often only slight in the border zone itself. On the other hand, we have not found a previous report of a fibroma of the lung with this appearance. Fibromata of the lung are rather rare, and those described are generally made up of typical compact fibrous tumours. In 1924 Sutherland, from the Mayo Clinic, reported a case of myxoma of the lung. The patient was a woman aged 41 years, who, eight years before admission to hospital, had been told that she had a lesion in the right apex. During the three years before admission she had had pain in the right arm and under the right scapula, and an enlargement on the right side of her neck had developed during the same period. She had lost $18 \frac{1}{2} \mathrm{lb}$. in weight during the previous 18 months. Examination revealed dullness to percussion, decreased respiration, and increased fremitus at the right apex. Radiography showed a circumscribed area of increased density in the upper right thorax. At operation a solid fibrous tumour was found. It was not adherent to the thoracic wall or the mediastinum, but was adherent to the lung in the centre of the thorax and was continuous with the swelling in the neck and over the clavicle. Microscopic examination showed it to be a myxoma, which was not removed; the patient was referred for radiotherapy. This is the only case of myxoma of the lung that has been described, and neither the clinical course nor the radiographic appearances resemble the lesion described in this report.

\section{SUMmaRY}

A case of chronic lung disorder, possibly a fibroma with inflammatory changes, which presented clinically and radiologically as a cystic lung, is reported. So far as can be ascertained, no similar case has previously been described.

REFERENCE

Sutherland, C. G. (1924). Radiology, 3, 161 . 
BIBLIOGRAPHY

Ball, H. A. (193!). Amer. J. Cancer, 15, 2319.

Johns, E. P., and Sharpe, W. C. (1935). Ibid , 23, 45

Lyssunkin, I. I. (1934). Frarkfurt Z. Path., 46, 007.

Scheidegger, S (:932). Z. Krebsforsch, 35, 172

Thibaudeau, A. A., and Kress, L. C. (1935). Amer. J. Cancer, 23, 267. 